\title{
A technique for optimizing ultrasonography-guided radial arterial catheter insertion
}

\author{
Satoru Fujii, MD (1) • Philip M. Jones, MD
}

Received: 5 February 2017/Revised: 9 February 2017/Accepted: 17 February 2017/Published online: 27 February 2017

(c) Canadian Anesthesiologists' Society 2017

\section{To the Editor,}

Using dynamic ultrasonography (US) to guide insertion of central venous catheters has become a standard of care as the technique is associated with improved safety and a better cannulation success rate. ${ }^{1}$ Despite a meta-analysis demonstrating reduced procedure time and reduced complications with the use of $\mathrm{US}^{2}$ for radial arterial catheterization, however, many clinicians still prefer a blind palpation-only technique over the use of US. This choice is partly because many clinicians struggle with a US-guided technique due to technical limitations imparted by the small diameter of the radial artery and because some studies have not demonstrated that the US-guided technique offers superior results (at least among experienced operators). ${ }^{3}$ We speculate, however, that it may also be because a practical step-by-step guide for its optimal use has not yet been published. According to the above-cited meta-analysis, ${ }^{2}$ first-attempt success rates using the US-guided technique range from $53 \%$ to $95 \%$. Although these numbers seem reasonable, they are probably not high enough to convince many anesthesiologists to convert to the US-guided technique that many find logistically cumbersome.

Electronic supplementary material The online version of this article (doi:10.1007/s12630-017-0850-z) contains supplementary material, which is available to authorized users.

S. Fujii, MD $(\bowtie) \cdot$ P. M. Jones, MD

Department of Anesthesiology, London Health Sciences Centre, University of Western Ontario, London, ON, Canada

e-mail: satoru.fujii@lhsc.on.ca

P. M. Jones, MD

Department of Epidemiology \& Biostatistics, London Health Sciences Centre, University of Western Ontario, London, ON, Canada
Recently, we developed an arterial catheter insertion technique using US guidance that we think will result in a higher first-attempt success rate. We herein report this insertion technique (along with a representative video clip [available online as Electronic Supplementary Material; ESM], for which the patient provided written consent to use in this report).

We use an L15-7io linear probe and the iE33 US device (Philips, Andover, MA, USA). First, we position the US monitor so it directly faces the operator. Using sterile technique, the US probe is used to visualize the radial artery at the centre of the screen, after which $1-2 \mathrm{~mL} 1 \%$ lidocaine is injected. The tip of the catheter needle is then inserted so it is on top of the radial artery. We advance the catheter at a shallow angle, visualizing the tip of the needle approximately $2-5 \mathrm{~mm}$ below the skin surface on the screen. An important caveat is that the radial artery is usually located about $5 \mathrm{~mm}$ below the skin, so if the angle of insertion is extremely acute, the needle tip can be easily advanced deeply, beyond the radial artery.

After confirming that the needle tip is on top of the radial artery, we angle the probe forward (i.e., away from the operator) until the needle tip disappears from the screen (because the needle tip is now out-of-plane with the US beam). When the needle tip disappears, we advance the needle until the tip reappears on top of the radial artery but at a deeper location. By iteratively repeating the same maneuver, we are able to feel the catheter enter the radial artery with a "pop" sensation. After the catheter enters the radial artery, the tip of the needle can clearly be seen within the radial artery. We then continue to tilt the probe dynamically and advance the needle slightly until the tip reappears - always being sure to keep the tip of the needle at the centre of the radial artery (i.e., in the centre of the bullseye). It requires that the operator use a progressively 
Figure Still frame image of a video available online (available as electronic supplemental material) shows cannulation of the radial artery. In image $\mathrm{A}$, the operator's hand position is clearly seen, and the angulation of the ultrasonography (US) probe away from the operator is noted. In image B, the catheter/ needle is seen within the "bullseye" of the radial artery image on the US console's display
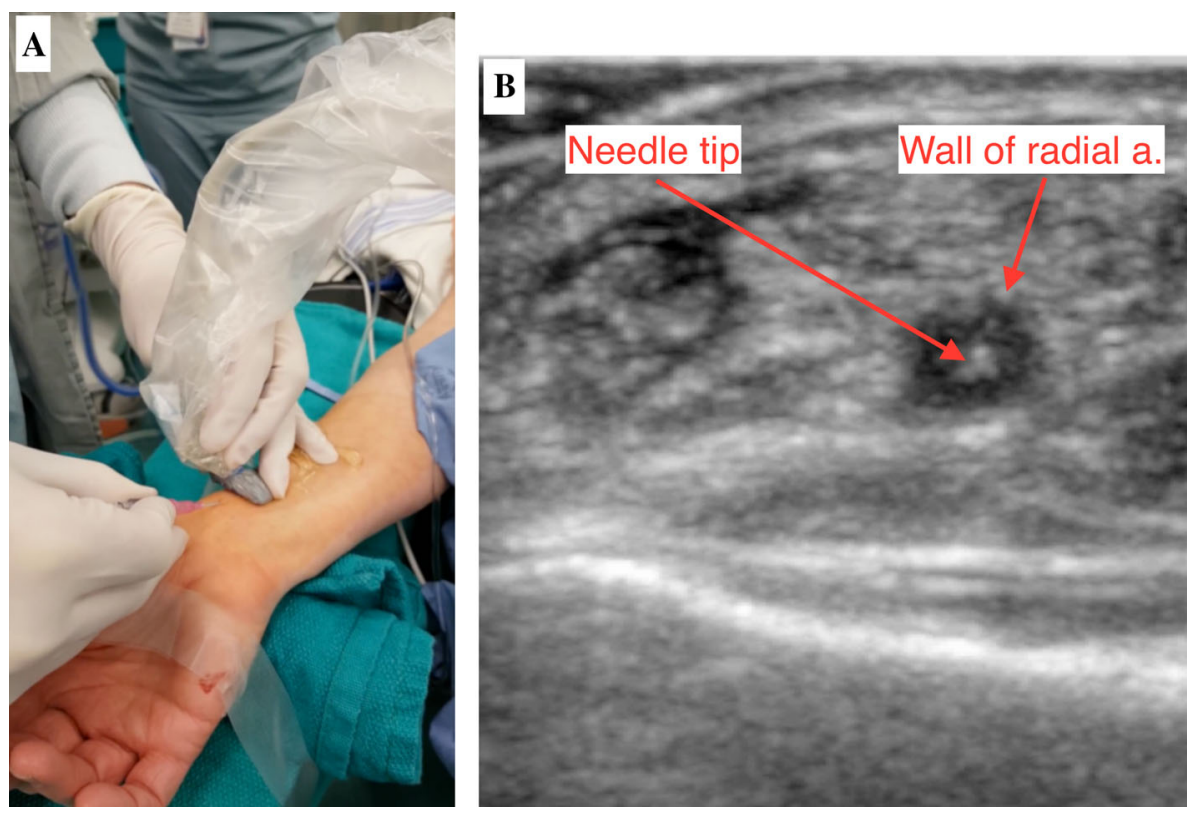

more shallow angle of the needle/catheter combination (see ESM video). When the catheter is well into the artery, it is advanced off the needle using a slight rotary motion.

There are a few keys to ensuring success with this technique. It is important to focus one's attention on the screen, not on the puncture site. Also, one must not attempt to thread the catheter the instant that the bloody flashback appears in the catheter hub. Instead, it is important to keep advancing the needle/catheter unit as one until it is seen (visualized as two parallel lines) on the US monitor screen. Although technical results will clearly vary according to practitioner, we have experienced a nearly $100 \%$ success rate using this technique.

Conflicts of interest None declared.
Editorial responsibility This submission was handled by Dr. Hilary P. Grocott, Editor-in-Chief, Canadian Journal of Anesthesia.

Funding None.

\section{References}

1. Reusz G, Csomos A. The role of ultrasound guidance for vascular access. Curr Opin Anesthesiol 2015; 28: 710-6.

2. White L, Halpin A, Turner M, Wallace L. Ultrasound-guided radial artery cannulation in adult and paediatric populations: a systematic review and meta-analysis. Br J Anaesth 2016; 116: 610-7.

3. Peters C, Schwarz SK, Yarnold CH, Kojic K, Kojic S, Head SJ. Ultrasound guidance versus direct palpation for radial artery catheterization by expert operators: a randomized trial among Canadian cardiac anesthesiologists. Can J Anesth 2015; 62: 1161-8. 